\title{
Effects of Devoflurane and Sevoflurane on Cognitive Function in Elderly Patients Undergoing General Anesthesia
}

\author{
Chunlu Zhang ${ }^{1}$, Bin Wang ${ }^{1}$, Jiali Ding ${ }^{1}$ and Peng Chen ${ }^{1, a}$ \\ ${ }^{1}$ Department of Anesthesiology, China-Japan Union Hospital, Ji Lin University, Changchun, China
}

\begin{abstract}
Objective: To compare effects of desflurane and sevoflurane on cognitive function in elderly patients undergoing general anesthesia. Methods Eighty elderly patients undergoing general anesthesia under open surgery were enrolled in this study, ASA I $\sim$ II, were divided into group A (desflurane group) and group B (sevoflurane group) randomly. Intravenous drugs included midazolam $0.03 \mathrm{mg} / \mathrm{kg}$, sufentanil $0.5 \mathrm{ug} / \mathrm{kg}$, propofol $1-2 \mathrm{mg} / \mathrm{kg}$, vecuronium 0.1 $\mathrm{mg} / \mathrm{kg}$. All patients were treated with desflurane or sevoflurane to maintain anesthesia, with depth 1.2 1.4MAC. The mini-mental state examination (MMSE) scores were recorded before operation and $1 \mathrm{~h}, 6 \mathrm{~h}, 12 \mathrm{~h}, 24 \mathrm{~h}$ and $48 \mathrm{~h}$ after operation. Results The MMSE scores of the two groups had no significant difference before operation but were significantly lower than those before operation $(\mathrm{P}<0.05)$. The MMSE scores of patients in group recovered at $12 \mathrm{~h}$ after operation $(\mathrm{P}<0.05)$. Scores of group B returned in $24 \mathrm{~h}$ after operation. Scores of group A were higher than group B at $1 \mathrm{~h}, 6 \mathrm{~h}$ and $12 \mathrm{~h}$ after operation, the difference was statistically significant. Conclusion The recovery time of cognitive function in elderly desflurane anesthesia patients was significantly less than that of sevoflurane.
\end{abstract}

\section{Background}

Postoperative cognitive dysfunction(POCD) is common in elderly patients undergoing complex surgery or emergency surgery, can be expressed as abnormal cognitive function, memory impairment or decline, personality and social integration capacity changes. The current reason was divers and no clear pathogenesis. At present, it argue that which method and drugs should be used in elderly patients maintenance of anesthesia ${ }^{\text {in }}$. Desflurane as the current blood gas partition coefficient of the smallest inhalation anesthetics, controllability is strong. At the same time, desflurane oil / gas partition coefficient is 18.7 , low blood tissue solubility ${ }^{21}$, the clinical manifestations of desflurane anesthesia induction and recovery are more rapid. The study of effect on desflurane anesthesia on the cognitive function of elderly patients is less ${ }^{\text {si. }}$. The aim of this study was to compare the effect of maintenance of anesthesia with desflurane or sevoflurane on the recovery of cognitive function in elderly patients, and to provide reference for clinical work.

\section{Materials and methods}

\subsection{General Information}

\footnotetext{
${ }^{\text {a }}$ Corresponding author: chenpeng5481@sina.com
} 
This study was approved by the ethics committee and signed informed consent with the patient. Eighty patients underwent abdominal surgery. American Society of Anesthesiologists (ASA) graded I-II, 41 males and 39 females, aged 60-85 years, with normal body mass index. The commonly used method for the study of POCD in the elderly was the most influential method ${ }^{\text {ta }}$, the MMSE scale, to assess the degree of cognitive function change after anesthesia ${ }^{\text {ss. }}$. Inclusion criterias were education level are high school and above, no liver,no kidney dysfunction (except for the need to treat the disease), 25 points or more MMSE score. Exclusion criterias: A.have severe visual and hearing impairment B.have a history of hypoxemia or asthma C. have a history of long-term smoking or alcohol abuse. D have cognitive dysfunction before surgery E cognitive impairment. F MMSE score $<25$ points. The patients were divided into two groups randomly.Both group had 40 patients, group A (desflurane group) B group (sevoflurane group). There were no significant differences in gender, age, body weight and BMI between two groups. See Table 1.

Table 1. two groups of patients with gender, age, body weight, BMI $(n=80, x \pm s)$

\begin{tabular}{ccccc}
\hline Number of groups & Male / Female $(\mathrm{n})$ & Age (years) & Weight $(\mathrm{Kg})$ & BMI \\
\hline Desflurane group & $19 / 21$ & $72.13 \pm 8.59$ & $59.40 \pm 8.10$ & $22.55 \pm 2.27$ \\
\hline Sevoflurane group & $22 / 18$ & $71.42 \pm 9.78$ & $58.53 \pm 6.97$ & $24.07 \pm 2.85$ \\
\hline
\end{tabular}

\subsection{Anesthesia method}

Heart rate(HR), blood oxygen saturation ( $\mathrm{SpO} 2)$, blood pressure and electrocardiogram (ECG) were measured and established venous access when went in the operation room. Anesthesia was induced midazolam $0.03 \mathrm{mg} / \mathrm{kg}$, sufentanil $0.5 \mu \mathrm{g} / \mathrm{kg}$, propofol $1-2 \mathrm{mg} / \mathrm{kg}$, vecuronium $0.1 \mathrm{mg} / \mathrm{kg}$, mechanical ventilation after intubation, $(8-10) \mathrm{ml} / \mathrm{kg}$, maintaining the end-tidal carbon dioxide partial pressure $\left(\mathrm{PctC}_{2}\right)$ between 35-45. Anesthesia maintenance used inhalation totally, groupA of inhaled desflurane,groupB inhalation of sevoflurane, MAC of $1.2 \sim 1.4$ during the surgery. Adjusted the MAC of not less than 0.820 minutes before the end of the operation ,.At the end of surgery, stopped inhalation of anesthetic, the oxygen flow to $5 \mathrm{~L} / \mathrm{min}$, when MAC was 0.2 or less and spontaneous breathing $\mathrm{Of}$ the $1 \mathrm{mg}$ plus atropine $0.5 \mathrm{mg}$ antagonistic residual muscle relaxation. When the patient returned to spontaneous breathing, called the patient $1 / \mathrm{min}$ times, until patients clearly, removed the tracheal tube after the recovery of reflex activity. After the patient returned to the ICU, recorded MMSE scores at1, 6, 12, 24, 48 h by anesthesiologists.

\subsection{Monitoring indicators}

The mean arterial pressure (MAP) of T1 (anesthesia initiation), T2 (intubation), T3 (surgical incision of skin), T4 (surgical end sutured skin) and T5 (extubation) were monitored during operation. Heart rate $(\mathrm{HR})$, electrocardiogram $(\mathrm{ECG})$, oxygen saturation $\left(\mathrm{SP}_{2}\right)$ and end-tidal carbon dioxide partial pressure $\left(\mathrm{PETCO}_{2}\right)$ were observed. The changes of cognitive function MMSE (Mini Mental State) were observed at 1, 6, 12, 24 and $48 \mathrm{~h}$ after operation. Recorded recovery period of restlessness, drowsiness, nausea, vomiting, respiratory depression and other adverse reactions.

\subsection{Statistical methods}

SPSS17.0 statistical software was used for data processing. Normal distribution of measurement data to mean \pm standard deviation (X soil $S$ ) table is used to compare between groups $t$ test, count data using repeated measurement of variance analysis, $\mathrm{P}<0.05$ for the difference was statistically significant.

A. Recovery times when from the end of surgery to awake group A was $8.2 \pm 4.6 \mathrm{~min}$, groupB was $12.3 \pm 5.0 \mathrm{~min}$, two groups were significantly different $(\mathrm{P}<0.05)$.

B. The comparison of MAP and HR between two groups of patients at different time points T1 
(anesthesia), T2 (intubation), T3 (surgical incision of the skin), T4 (surgical closure of the skin), T5 (extubation), $(\mathrm{P}>0.05)$, there were no difference at same time point . MAP and HR at each time point and no significant difference compared before anesthesia $(\mathrm{P}>0.05)$ Table 2 and Table 3.

Table 2. two groups of patients MAP ( $\mathrm{mmHg})$ comparison $(\mathrm{n}=40, \mathrm{x} \pm \mathrm{s})$

\begin{tabular}{cccccc}
\hline Group & T1 & T2 & T3 & T4 & T5 \\
\hline Group A & $89 \pm 21$ & $86 \pm 18$ & $94 \pm 27$ & $106 \pm 18$ & $90 \pm 25$ \\
\hline Group B & $85 \pm 17$ & $88 \pm 22$ & $96 \pm 23$ & $100 \pm 11$ & $95 \pm 13$ \\
\hline
\end{tabular}

Table 3. two groups of patients HR (times / min) comparison $(n=40$, x soil s)

\begin{tabular}{cccccc}
\hline Group & T1 & T2 & T3 & T4 & T5 \\
\hline Group A & $78 \pm 11$ & $93 \pm 9$ & $90 \pm 10$ & $91 \pm 8$ & $85 \pm 13$ \\
\hline Group B & $80 \pm 8$ & $88 \pm 11$ & $92 \pm 11$ & $93 \pm 7$ & $90 \pm 11$ \\
\hline
\end{tabular}

C. There was no significant difference in MMSE score between the two groups before operation (P> 0.05). MMSE scores of the two groups were significantly lower than those before operation $(\mathrm{P}<0.05)$ at $1 \mathrm{~h}, 6 \mathrm{~h}$ and $12 \mathrm{~h}$ after operation, The score of MMSE in group A decreased significantly $(\mathrm{P}<0.05)$ at $1 \mathrm{~h}$ and $6 \mathrm{~h}$ after operation, and returned to the preoperative level at $12 \mathrm{~h}$ after operation. The MMSE scores of group B were significantly lower than those before operation $(\mathrm{P}<0.05)$ at $1 \mathrm{~h}, 6 \mathrm{~h}$ and $12 \mathrm{~h}$ after operation, and returned to preoperative level $24 \mathrm{~h}$ after operation. The MMSE scores of group A were higher than those of group B $(\mathrm{P}<0.05)$. See Table 4 .

Table 4. comparions of MMSE scores changes on two groups of patients before and after operation

\begin{tabular}{ccccccc}
\hline Group & Preoperative & $\begin{array}{c}\text { 1h after } \\
\text { operation }\end{array}$ & $\begin{array}{c}\text { 6h after } \\
\text { operation }\end{array}$ & $\begin{array}{c}12 \mathrm{~h} \text { after } \\
\text { operation }\end{array}$ & $\begin{array}{c}24 \mathrm{~h} \text { after } \\
\text { operation }\end{array}$ & $\begin{array}{c}48 \mathrm{~h} \text { after } \\
\text { operation }\end{array}$ \\
\hline Group A & 29.5 土 0.5 & 26.7 土 $1.1^{\star \star}$ & 28.4 土 $1.4^{\star \star}$ & 29.1 土 0.6 & 29.4 土 1.0 & 29.6 土 0.4 \\
\hline Group B & 29.6 土 0.6 & 24.7 土 $1.3^{\star \star}$ & 26.9 土 $0.7^{\star \star}$ & 27.9 土 $1.4^{\star}$ & 29.1 土 1.0 & 29.5 土 0.3 \\
\hline
\end{tabular}

* Comparison between groups $\mathrm{P}<0.05 \quad \star$ Comparison within the group $\mathrm{P}<0.05$

D. Comparison of adverse reactions between the two groups of nausea and vomiting and other side effects were no significant difference $(\mathrm{P}>0.05)$.

\section{Discussion}

Postoperative cognitive dysfunction manifest as memory impairment, visual and auditory impairment, and social behavior disorder, but also for anxiety, agitation, depression and other behavior changes. The current studies show that surgery and general anesthesia will affect the cognitive function of elderly patients significantly ${ }^{6}{ }^{6}$, furthermore a small number of patients still exist cognitive dysfunction after a long time. At present, the etiology and pathogeny of POCD have not been clarified. However, studies at home and abroad have shown that it is clear that anesthesia drugs and aging are the cause of POCD $^{7}$. So how to avoid the occurrence of POCD in clinical work is great significancely. Desflurane is a new halogen inhalation anesthetic drug, characterized by low blood gas partition coefficient and low blood tissue solubility, which can adjust the depth of anesthesia rapidly, induction of anesthesia quickly and rapid recovery ${ }^{8}$. We used MMSE score criteria which widely used and most influencely at home and abroad to determine the postoperative cognitive function, the use of desflurane or sevoflurane to maintain anesthesia depth of elderly patients after surgery and evaluate the advantages and disadvantages of two inhaled anesthesia in elderly patients with postoperative cognitive function.

The results showed that there was no significant difference in MMSE scores between two groups before operation. The MMSE scores of the two groups were significantly lower than the preoperative one. The decrease was more than 2 points, this shows that the two groups of patients were effected by maintenance of inhaled anesthesia on cognitive function short-term. This may be due to the decrease in the brain's reserve capacity in the elders. Inhaled anesthetic drugs may interfere with neurons by antagonizing NMDAR (N-methyl-D-aspartate receptors) and exciting gamma-aminobutyric acid 
receptors (GABAAR). And synaptic development of the environment, the role of induced neurotoxicity, leading to apoptosis of brain cells, affecting synaptic plasticity, and ultimately cause cognitive impairment. At the same time hemodynamic parameters experimental were no significant differences in two groups of patients, which also ruled out hypotension, hypoxia and other factors cause POCD. Importantly, the results also showed that the MMSE scores returned to preoperative levels at 12 hours after surgery in group A, and the MMSE score returned to preoperative levels more than 12 hours in group B. At the same time, the score of MMSE in group A was significantly higher than that in group B at 1 hour and 6 hours after operation. This shows it is desflurane that the change of patient's postoperative cognitive function may be mild, and the recovery quickly. The time when all patients awaked after operations in group A were shorter than group B, which also confirmed the results indirectly. The reason may be that desflurane has a low blood gas partition coefficient and tissue dissolution rate, anesthesia induction and recovery rapid, drug uptake and release rapidly, and low pharmacokinetics in vivo. These pharmacological features include NMDAR (N-methyl- Aspartate receptor) and excitatory gamma-amino butyric acid receptor (GABAAR) receptors make it reality that have less effect on postoperative cognitive function than sevoflurane. At the same time, desflurane anesthesia wake up faster than sevoflurane anesthesia patients, it is a reason that the patient recover preoperative levels on the aspect of work and rest time confusion, the psychological and so on.

The results of this study show that the use of two inhaled anesthetics, the elders have a short period of postoperative cognitive dysfunction, but the anesthesia of desflurane anesthesia have less affect on cognitive function than sevofluran and recover faster.

\section{References}

1. Harman D. Aging:overview [J].Ann N Y Acad Sci,2001,928:11-21.

2. Hangzhou 200127. Pharmacology and clinical application of J. Shanghai medical journal, 2010,4:298-303.

3. Chen Bin Zheng Hui. 3, research progress $\mathrm{J}$ cognitive dysfunction of inhalation anesthetic and postoperative. Medical review, 2013,10:1850-1852.

4. shows. The psychiatric rating scale manual $[\mathrm{M}] .2$ version. Changsha: Hunan science and Technology Press, 2003,33-39.

5. Wang Huiwen, Yang Lifang, Xiong et al. Different scale assessment after general anesthesia in elderly patients with early cognitive function compared J meaning anesth, 2001,3:459-461.

6. Cao Xiaoyang $\mathrm{J}$ observation of cognitive dysfunction after general anesthesia in elderly patients. Journal of medical forum, 2006,3:43-44.

7. horse Ya Bing. Different ways of anesthesia research J effects on cognitive function in elderly patients after surgery. China clinical medicine, 2014,11:26-28.

8. Fu Hui. J anesthesia effect on gynecological laparoscopic intrapulmonary shunt and blood gas. China medical science, 2013,20:16-17. 